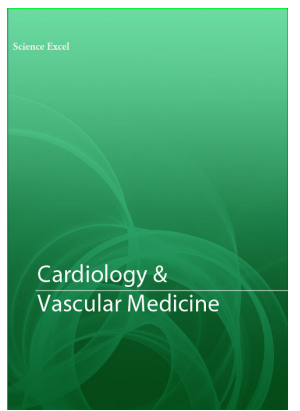

Correspondence

Greg Flaker M.D. FACC. FACP

CE 351 University Hospital CE351, Columbia, MO 65212, USA

Tel: + 1 (573) 884-2296

Fax: (573) 884-3221

E-mail: flakerg@health.missouri.edu

\footnotetext{
- Received Date: 01 Oct 2020;

- Accepted Date: 07 Oct 2020;

- Publication Date: 10 Oct 2020.
}

Abbreviations

AC: anticoagulation; VKA: vitamin $K$ antagonist; TE: thromboembolic event; UFH: unfractionated heparin; LMWH: low molecular weight heparin; TIA: transient ischemic attack; HIT: heparin induced thrombocytopenia; DOAC: direct acting oral anticoagulant: AHA/ACC/HRS: American Heart Association/American College of Cardiology/ Heart Rhythm Society; EHRA European Heart Rhythm Association; PAUSE: Perioperative Anticoagulation Use for Surgery Evaluation

\section{Copyright}

(C) 2020 Science Excel. This is an openaccess article distributed under the terms of the Creative Commons Attribution 4.0 International license.

\title{
Periprocedural Management of Anticoagulation in the Era of Direct Acting Oral Anticoagulants
}

\author{
Troy Loethen and Greg C Flaker \\ University of Missouri School of Medicine, Columbia, MO, USA
}

\section{Periprocedural Management of Anticoagulation with Vitamin K Antagonists}

Patients who receive oral anticoagulation (AC) for stroke prevention often undergo surgical procedures. The interruption of oral AC might result in a thromboembolic event (TE), especially in patients at higher risk for TE. To prevent TE, parenteral AC is often provided during this interruption of oral AC. This practice is referred to as "bridging anticoagulation."

Interruption of oral $\mathrm{AC}$ is common, occurring in up to $30 \%$ of patients over a 2 year period [1]. In the case of vitamin K antagonists (VKA) the interruption may result in sub-therapeutic anticoagulation for over 10 days. This is due to the fact that VKAs achieve their anticoagulant effect by synthesis of dysfunctional clotting factors. Because the half- lives of some of these endogenous factors are long, warfarin is often stopped for 5 days prior to surgery, to ensure adequate hemostasis. Because of the long halflives, when VKA's are re-started after surgery, it may take 4-5 days or longer to achieve therapeutic anticoagulation, usually defined as an INR $>2.0$. Traditionally, parenteral AC with either unfractionated heparin (UFH) or low molecular weight heparin (LMWH) can be used in the perioperative period to prevent a TE.

Unfortunately, the effectiveness of this bridging strategy has been difficult to prove. A large review in patients who required interruption of VKA's for surgical procedures showed no significant differences in TE between those patients who received parenteral AC and those who did not. More importantly, a significant increase in major bleeding was noted in patients receiving parenteral AC [2]. Additional prospective data showed similar results. The Bridging Anticoagulation in Patients who Require Temporary Interruption of Warfarin Therapy for an Elective Procedure or Surgery (BRIDGE) study involved patients who were at moderate risk for TE (mean $\mathrm{CHADS}_{2}$ score $=2.3$, scale 1-6 with higher scores indicating higher risk of TE) and who underwent surgery. Patients were randomized to receive either a $\mathrm{LMWH}$ (dalteparin) or placebo. The results showed that there were no significant differences in a low rate of TE between groups who received or did not receive bridging therapy. However, significantly higher rates of major bleeding were reported with dalteparin [3]. Whether or not patients at higher risk for TE would benefit from bridging anticoagulation is the subject of ongoing studies. Because of these data, strategies have evolved to improve perioperative care in anticoagulated patients. One of these strategies is to simply not interrupt oral AC, especially in surgical procedures thought to be a lower risk for bleeding. Studies showed that procedures including pacemaker and implantable cardioverterdefibrillator implantation, dental extraction, and cataract surgery could be performed safely without interruption of VKA [4-8]. Surgical procedures with lower, moderate and higher risk of bleeding have been published [9].

A second strategy is to evaluate the risk of $\mathrm{TE}$ in the patient undergoing surgery. If the patient is at low risk for a TE $(<5 \% /$ year), with a $\mathrm{CHA}_{2} \mathrm{DS}_{2}$-VASc score of $<4$ (score 1-9, higher score indicates higher risk) or no prior history of stroke or transient ischemic attack (TIA), and planned surgery is at higher risk for bleeding, surgery is performed after holding VKA for 5 days prior to surgery. VKA is restarted on the day of surgery when hemostasis is achieved. No parenteral AC is provided.

If the patient is at high risk for a TE (>10\%/year) including those with a $\mathrm{CHA}_{2} \mathrm{DS}_{2}$-VASc score of $>7$ or those with a recent history of stroke, TIA, or systemic embolism and planned surgery is at higher risk for bleeding, VKA is stopped 5 days prior to surgery. Two days after stopping VKA, or when the INR is $<2.0$, parenteral AC with UFH, LMWH, or other agents could be considered. The dose and duration of these agents vary from institution to institution and from different specialists with fixed, weight based doses and weight based initial dose 
followed by reduced dosed closer to surgery being described [3,10-13]. Some gastroenterologists might feel comfortable with performing endoscopy with biopsy while others will not. Similarly some interventional cardiologists will proceed with coronary angiography in selected patients of VKA, while others will insist on holding VKA for several days. This emphasizes the importance of a multi-disciplinary approach in the management of these patients [14].

The type of parenteral anticoagulation provided is also variable. $\mathrm{UFH}$ is considered in patients with poor renal function (eGFR $<30 \mathrm{cc} / \mathrm{min}$ ) or if the patient is uncomfortable with self- injection. $\mathrm{UFH}$ requires hospitalization. UFH is stopped $>4$ hours prior to the procedure and residual $\mathrm{AC}$ effect can be monitored by the partial thromboplastin time. If LMWH is used as parenteral AC, it is stopped at least 24 hours prior to the procedure. Anticoagulant effect can be measured by LMWH-specific antifactor Xa assay.

Re-initiation of VKA can usually be performed within 24 hours after the procedure and after hemostasis is achieved. Re-initiation of UFH or LMWH might be started within the first 24 hours but in selected patients might be delayed for 48-72 hours. UFH and LMWH is continued until the INR is $>2.0$ [15].

There are a number of cases in which the decision as to who and how to offer bridging anticoagulation is subject to considerable clinical judgement. Some surgical teams might perform surgeries at moderate risk with no interruption of parenteral AC. If a patient is at moderate risk for TE (5-10\%/year) with a CHA $\mathrm{DS}_{2}-$ VASc score of 5-6 or with a remote history ( $>3$ months) of stroke, TIA or systemic embolism, parenteral AC might not be offered if there is perceived risk of increase bleeding. However if the risk of bleeding is judged to be low, these same patients might be considered eligible for bridging.

Patients who have a history of heparin induced thrombocytopenia (HIT), in general, should not receive UFH or LMWH. For these patients who require parenteral AC, direct thrombin inhibitors such as argatroban or bivalirudin are options [16]. Experience with direct acting oral anticoagulants (DOAC) in patients with HIT has been reported [17], making this a possible option for bridging in the future.

Finally, the issue of appropriate parenteral AC for the patient with a mechanical heart valve should be addressed. At one point the Food and Drug Administration issued a black box warning stating that LMWH, specifically enoxaparin, was "not recommended for thromboprophylaxis in patients with prosthetic heart valves." Studies have shown the relative safety and effectiveness on LMWH in patients with mechanical heart valves [18,19]. Given the fact that LMWH can be given as an outpatient and avoid the hospital expense associated with UFH [20], local protocols can be justified which incorporate LMWH is selected patients with mechanical valves. For example, if a patient with a mechanical heart valve undergoes surgery with a low bleeding risk, it is reasonable to not interrupt VKA. If the risk of surgical bleeding requires interruption of $\mathrm{VKA}$, an assessment of the TE risk is made. If the patient is at low risk for TE (mechanical bi-leaflet aortic valve, sinus rhythm), it would be reasonable to avoid preoperative parenteral AC. If the postoperative recovery is brief, post-operative AC could be avoided. If the patient is a moderate or higher risk for TE (mechanical mitral valve), preoperative and postoperative LMWH could be considered [21]. Although LMWH has not been studied adequately in patients with mechanical prosthetic valves, particularly those on dialysis, doses of $0.4-1 \mathrm{mg} /$ $\mathrm{kg}$ /day have been used to achieve therapeutic anticoagulation in hemodialysis patients and with comparable safety to UFH [22].

\section{Periprocedural Management of Anticoagulation with Direct Acting Oral Anticoagulants}

In comparison to VKA, the periprocedural management DOACs in planned procedures has not been as well understood and controversy remains in clinical practice since their introduction. To address this lack of comprehensive data and to supplement the American College of Cardiology Expert Consensus Statement in 2017, the European Heart Rhythm Association (EHRA) provided practical suggestions on the use of DOACs in patients with AF.

EHRA categorized patients as either low or high bleeding risk procedures. EHRA recommends no interruption of DOACs in minor-bleeding risk procedures, omitting DOACs 24 hours before and after the procedure in low-risk bleeding procedures, and omitting DOACs 48 hours before and after the procedure in high-risk bleeding procedures. In high-bleeding risk procedures, DOAC plasma levels may be considered to guide procedure timing. EHRA warns, however, this is not evidence-based management and safe DOAC levels is not well established. EHRA also does not recommend bridging with $\mathrm{LMWH}$ or heparin in patients taking DOACs.

To provide prospective data on the timing of DOAC interruption and resumption, the Perioperative Anticoagulation Use for Surgery Evaluation (PAUSE) study was performed [23].

The PAUSE study was a prospective study which used a simple standardized strategy for interruption and resumption of DOACs without bridging therapy in 3,007 patients with AF or venous TE undergoing elective procedures or surgery requiring AC interruption. Patient were categorized as either having a low or high bleeding risk procedure. DOACs studied included apixaban, dabigatran, and rivaroxaban. For low-risk bleeding procedures, DOACs were omitted 24 hours before and after the procedure. For high-risk bleeding procedures, DOACs were omitted 48 hours before and after the procedure. Residual anticoagulant levels were obtained just before the procedure, which showed that $>90 \%$ of patients had a minimal residual level $(<50 \mathrm{ng} / \mathrm{ml})$ of anticoagulant at time of the procedure. The study showed that the 30 day risk of major bleeding $(<2 \%)$ and arterial TE $(<1 \%)$ were low with the proposed DOAC periprocedural strategy [24].

Edoxaban, a fourth DOAC, has also been used safely and effectively with preliminary results showing low rates of major bleeding (0.7\%), non-major bleeding (1.4\%), and thrombotic/ ischemic events $(0.6 \%)$ [25].

These updated guidelines and findings will help give clinicians more confidence to manage DOACS in the perioperative setting of planned procedures and gives some standardization to management.

\section{Conflict of interest statement}

The authors have no conflicts of interests to declare..

\section{References}

1. Steinberg BA, Peterson ED, Kim S, et al. on behalf of the Outcomes Registry for Better Informed Treatment of Atrial Fibrillation (ORBIT-AF Investigators. Use and outcomes associated with bridging during anticoagulation interruptions in patients with atrial fibrillation. Circulation. 2015;131:488494.

2. Siegal D, Yudin J, Kaatz S, et al. Periprocedural heparin bridging in patients receiving vitamin $\mathrm{K}$ antagonists: systematic review and meta-analysis of bleeding and thromboembolic rates. Circulation. 2012;126:1630-1639. 
3. Douketis JD, Spyropoulos AC, Kaatz S, et al. Perioperative bridging anticoagulation in patients with atrial fibrillation. $\mathrm{N}$ Engl J Med. 2015;373:823-833.

4. Birnie DH, Healey JS, Wells GA, et al. Pacemaker or defibrillator surgery without interruption of anticoagulation. N Engl J Med. 268;22:2084-2093.

5. Coyle D, Coyle K, Essebag V, et al. Cost effectiveness of continued- warfarin versus haparin-bringing therapy during pacemaker and defibrillator surgery. J Am Coll Cardiol. 2015;65:957.

6. Cheng A, Nazarian S, Brinker JA, et al. Continuation of warfarin during pacemaker or implantable cardioverter-defibrillator implantation: a randomized clinical trial. Heart Rhythm. 2011;8:536-540.

7. Bajkin BV, Popovic SL, Selakovic SD. Randomized, prospective trial comparing bridging therapy using low-molecular-weight heparin with maintenance of oral anticoagulation during extraction of teeth. J Oral Maxillfac Surg. 2009;67:990-995.

8. Katz J, Feldman MA, Bass EB, et al., Study of medical testing for cataract surgery team. Risks and benefits of anticoagulant and antiplatelet medication use before cataract surgery. Ophthalmology. 2003;110:1784-1788.

9. Heidbuchel H, Verhamme P, Alings M, et al. European heart rhythm association practical guide on the use of new oral anticoagulants in patients with non-valvular atrial fibrillation. Europace. 2013;15:625-651

10. Pengo V, Cucchini U, Denas G, et al. Standardized lowmolecular-weight heparin bridging regimen in outpatients on oral anticoagulants undergoing invasive procedure. Oral Surgery. 2009;119:2920-2927.

11. Rechenmacher S, Fang JC. Bridging Anticoagulation J Am Coll Cardiol. 2015;66:1392-403.

12. Kovacs MJ, Kearon C, Rodger M, Anderson DR. Single-arm study of bridging therapy with low-molecular-weight heparin for patients at risk of arterial embolism who require temporary interruption of warfarin. Circulation. 2004;110:1658-1663.

13. Wysokinski W, McBane R II. Periprocedural bridging management of anticoagulation. Circulation 2012;126:486-490.

14. Flaker G, Theriot P, Binder LG, Dobesh PP, Cuker A, Doherty JU. A survey of the management of periprocedural anticoagulation in contemporary practice. JACC. 2016;68:217-226.
15. Doherty JU, Gluckman TJ, Hucker WJ, et al. 2017 Expert consensus decision pathway for periprocedural management of anticoagulation in patients with nonvalvular atrial fibrillation. JACC. 2017;69(7):871-898.

16. Skrupky LP, Smith JR, Deal EN, et al. Comparison of bivalirudin and argatroban for the management of heparin-induced thrombocytopenia. Pharmacotherapy. 2010;12:1229-38.

17. Warkentia TE, Pai M, Linkins LA. Direct oral anticoagulants for treatment of HIT: update of Hamilton experience and literature review. Blood. 2017;130:1104.

18. Meurin P, Tabet JY, Weber H, Renaud N, Ben Driss A. Lowmolecular-weight heparin as bridging anticoagulant early after mechanical heart valve replacement. Circulation. 2006;113:56469.

19. Montalescot G, Polle V, Collet JP, et al. Low molecular weight heparin after mechanical heart valve replacement. Circulation. 2000;101:1083-86

20. Amorosi SL, Tsilimingras K, Thompson D, Fanikos J, Weinstein MC, Goldhaber SZ. Cost analysis of "bridging therapy" with low-molecular-weight heparin versus unfractionated heparin during temporary interruption of chronic anticoagulation. Am J Cardiol. 2004;93(4):509-511.

21. Tan CW, Wall M, Rosengart TK, Ghanta RK. How to bridge? Management of anticoagulation in patients with mechanical heart valve undergoing noncardiac surgery. J Thorac Cardiovasc Surg. 2019;158:200-203.

22. Pon TK, Dager WE, Roberts AJ, White RH. Subcutaneous enoxaparin for therapeutic anticoagulation in hemodialysis patients. Thrombosis Research. 2014;133:1023-28.

23. Steffel J, Verhamme P, Potpara TS, et al. The 2018 European Heart Rhythm Association Practical Guide on the use of nonvitamin $\mathrm{K}$ antagonist oral anticoagulants in patients with atrial fibrillation. Eur Heart J. 2018;39(16):1330-1393.

24. Douketis JD, Spyropoulos AC, Duncan J, et al. Perioperative management of patients with atrial fibrillation receiving a direct oral anticoagulant. JAMA Intern Med. 2019;179(11):1469-1478.

25. Colonna P, von Heymann C, Santamaria A, Matsushita Y, Unverdorben $M$. Edoxaban management in diagnostic and therapeutic procedures (EMIT-AF/VTE)-Trial design. Clin Cardiol. 2018;41(9):1123-1129. 